\title{
Research of the spectral characteristics of healthy and fusarium-infected oat seeds of the variety «ZALP» with hyperspectral camera
}

\author{
Maksim Moskovskiy ${ }^{1,}$, Stanislav Gerasimenko ${ }^{1}$, Andrey Boiko ${ }^{2}$ and Sergey Vorobev ${ }^{2}$ \\ ${ }^{1}$ Federal Scientific Agro-Engineering Center VIM, 1 Institutsky proezd, 5, Moscow, 109428, Russia \\ ${ }^{2}$ Don state technical university, Gagarin Square, 1, Rostov-on-Don, 344000, Russia
}

\begin{abstract}
The paper presents the research of the spectral lines of healthy and fusarium-infected oat seeds using a hyperspectral imaging system. Hyperspectral images of healthy and diseased kernels were studied using the Specim IQ hyperspectral camera and Albedo 4.0.23 program (for subsequent processing). The spectral diagrams of the horizon $(800 \mathrm{~nm}-1000 \mathrm{~nm})$ have been determined. They can identify the oat seeds of the ZALP variety which infected with pathogenic microflora by fungi of the genus Fusarium. Variety harvested in the central zone of the Russian Federation.
\end{abstract}

\section{Introduction}

Important technology for non-invasive impact on seeds and monitoring the biological composition of seeds is remote sensing using hyperspectral cameras. This method is directly related to the physiological seeds state or state of infection with pathogens [1-4].

One of the popular cameras used in scientific research is the Specim IQ mobile hyperspectral camera. This camera was used by Russian scientists to research wheat seed infected by simple root rot and to analyze the pulp of potato tubers [5]. The paper presents the experience of using a hyperspectral camera to study the oats seeds the variety «ZALP» infected with fungi of the genus Fusarium (fusarium).

Fusarium head blight of cereals is one of the most common fungal diseases of grain and ears. The chemical method for preventing the development of mycotoxins of Fusarium fungi is not able to fully ensure control over the state of the plant ear the due to the limited set of fungicides and the short period of time of its application. Any batch of seeds in which Fusarium mycotoxins have been found are discarded and the batch becomes completely unfit for food use [6-7,10].

Seeds infected with Fusarium are outwardly pale pink, wrinkled and lightweight, lose germination or give weakened seedlings. With a weak lesion, seeds infected with Fusarium do not visually differ from healthy ones. The chemical composition of such seeds changes significantly, the protein decomposes with a noticeable release of ammonia. As a result, the seeds endosperm is friable, crumbly and the vitreousness is very low. The elasticity and swelling of the gluten also decreases. The main factor for determining the contamination of

*Corresponding author: maxmoskovsky74@yandex.ru 
grain with mycotoxins is the infection degree and the species composition of fungi of the genus Fusarium. The most famous groups of trichothecene mycotoxins are deoxynivalenol (DON) and zearalenone (ZEA), fumonisins [7].

In the Russian Federation, maximum permissible concentrations for the content of fusariotoxins have been established, for deoxynivalenol - 0.7-1 mg / kg, zearalenone - $5 \mathrm{mg}$ $/ \mathrm{kg}$, etc. [6-7].

The different degrees of Fusarium infestation on an oat ear presented on the Figure 1. To detect this diaphoretic disease, it makes sense to use a non-invasive method of examining seeds using hyperspectral cameras.

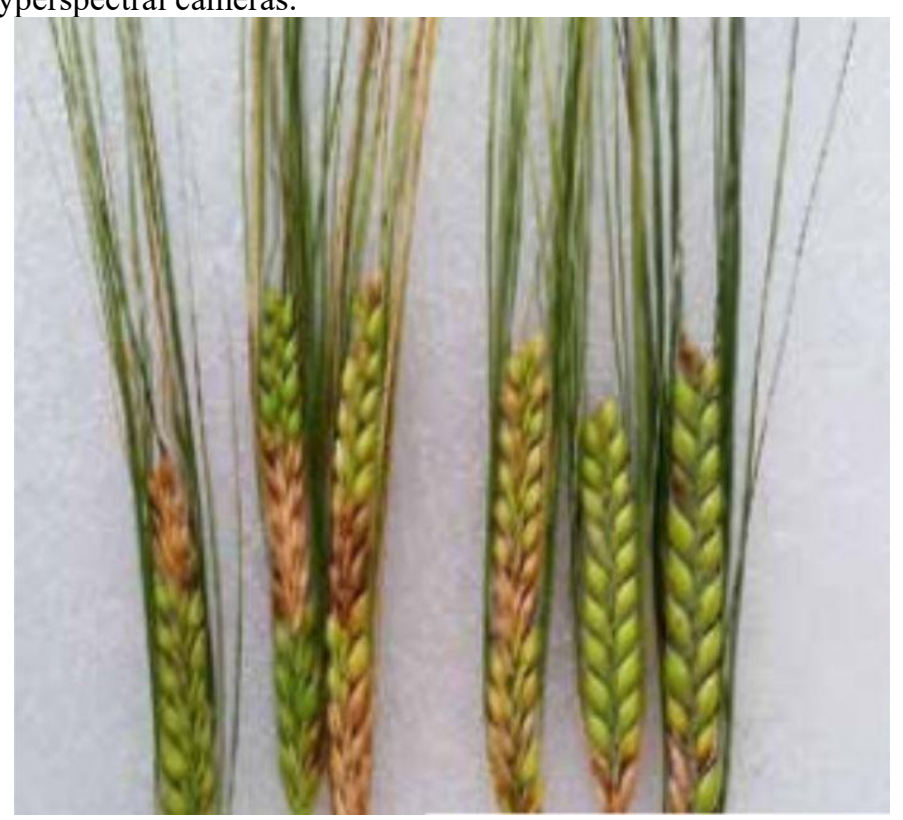

Fig. 1. External degree of infestation of an ear of oats with Fusarium

\section{Materials and methods}

\subsection{Samples of oat seeds of the variety «ZALP» for the study of fusarium infestation}

Oats variety «ZALP» were used as a starting material (Fig. 2). As can be seen from Figure 2 , there are no pronounced symptoms of pathogen infection on the infected seeds.

The most typical oat potagen is the T-2, HT-2-producing toxins and nivalenol. These information confirm the results of the monitoring of mycotoxins in the territory of the Russian Federation, carried out by the researches of All-Russian Research Institute of Veterinary Sanitation, Hygiene and Ecology (ARRIVSHE) [7]. A comparative analysis of the reflectivity of healthy and infected oat seeds with Fusarium was carried out. [4, 8]. 


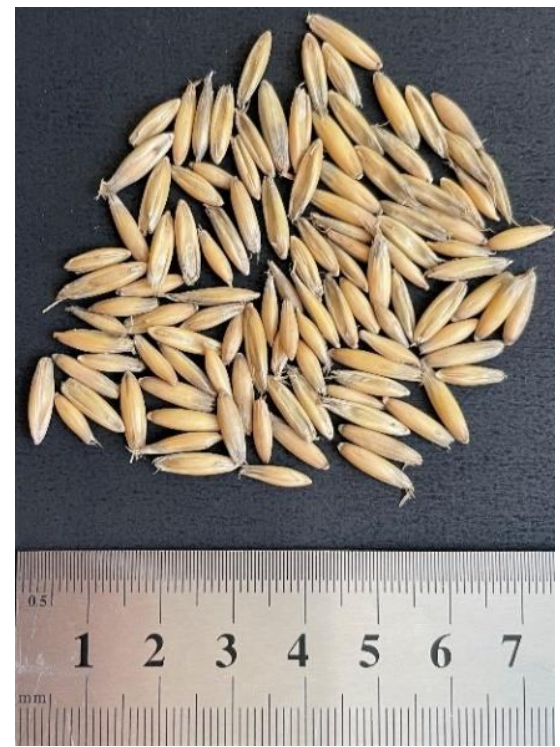

a)

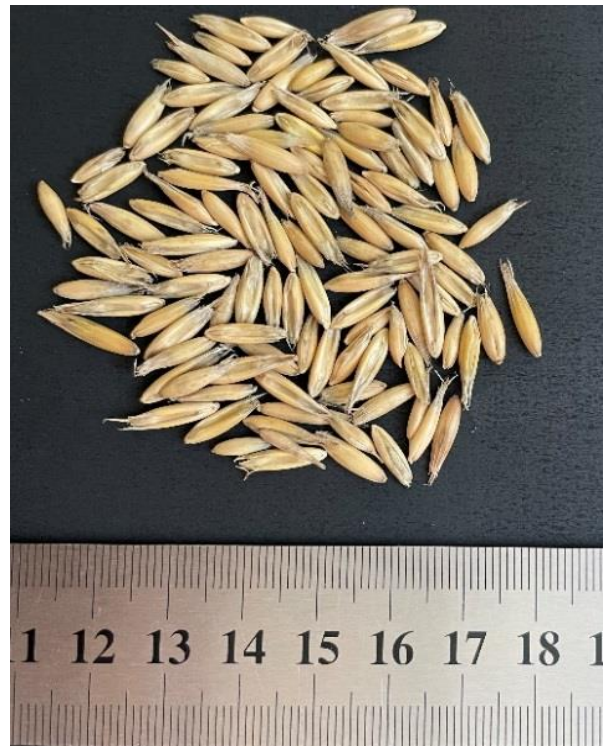

b)

Fig. 2. The starting material of oat variety «ZALP» a) Healthy seeds b) Seeds affected by fusarium

Spring oats of the variety «ZALP» ( mutika sort) have the following biological characteristics: white-grain, awnless, seed weight is $33-41 \mathrm{~g}$, the average yield is about 44.7 $\mathrm{c} / \mathrm{ha}$, the maximum yield was recorded $67.9-72.6 \mathrm{~kg} / \mathrm{ha}$ according to various sources, growing season is $69-90$ days, resistant to lodging and shedding, protein content is 11.1 $14.2 \%$, filminess is $26.7 \%$, grain nature is $380-510 \mathrm{~g} / 1$.

In field conditions, resistance to diseases of crown and stem rust was weakly affected, bacterial burns and reddish-brown spotting - medium. Early ripening and the vertical arrangement of the flag leaf favor mixed sowing with vetch for forage and seed purposes. Originator of FGBNU «Federal Research Center «NEMCHINOVKA», year of inclusion in the register 2015 .

The selected sample was the 2020 year harvest from the Central Black Earth zone of Russia. 6 seed samples 1000 grains each were taken as samples for the research. Three samples were infected with fusarium with varying degrees of damage of 50-72\% from the 6 samples.

\subsection{Equipment}

Specim IQ hyperspectral camera (Spectral Imaging Ltd., https://www.specim.fi/iq/) was used as the equipment for the research. The camera allows evaluating the reflection spectra in the range 400-1000 $\mathrm{nm}$. The spectral resolution of the camera is $7 \mathrm{~nm}$ and includes 204 bands, the spatial resolution of the sensor is $512 \times 512$ pixels. The camera was installed on a tripod above the table, at a distance of $15-20 \mathrm{~cm}$ from the sample, taking into account that the calibration plate does not occupy more than $10 \%$ of the image. The samples were illuminated with $72 \mathrm{~W}$ luminescent panels. Before shooting, the camera was calibrated using a calibration panel, after which, when receiving a series of images, the panel was removed from the frame. [9]. 


\subsection{Method of Spectral image processing}

Preliminary visual quality control of hyperspectral data was carried out using the Albedo 4.0.23 program (Moscow Institute of Physics and Technology, https://geo.mipt.ru/albedo/).

This program is intended to determine the physico-chemical composition of the object by the pixels of the raster image. Each pixel is characterized by its own radiation spectrum located in the range of $400-1000 \mathrm{~nm}$.

Each image is analyzed in the following specters: Near Infrared (NIR), Red, Green, Blue. Polygons on the healthy and contaminated oat samples are highlighted on the research images to exclude extraneous objects from the field of analysis (Fig. 3). The spectral line was calculated for each polygon.

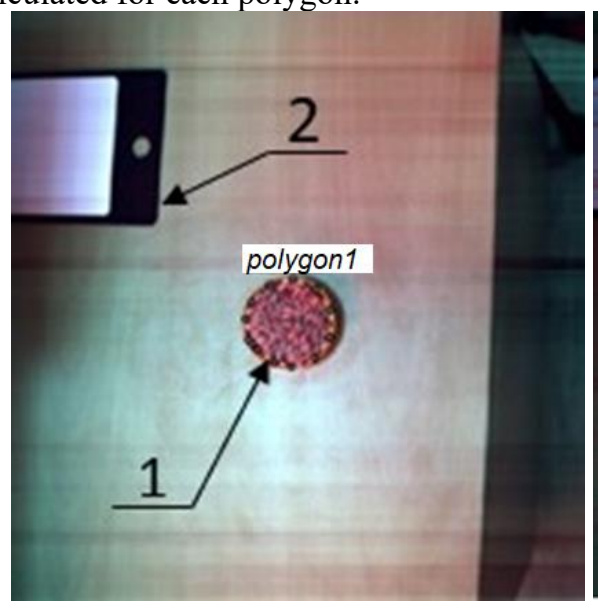

a)

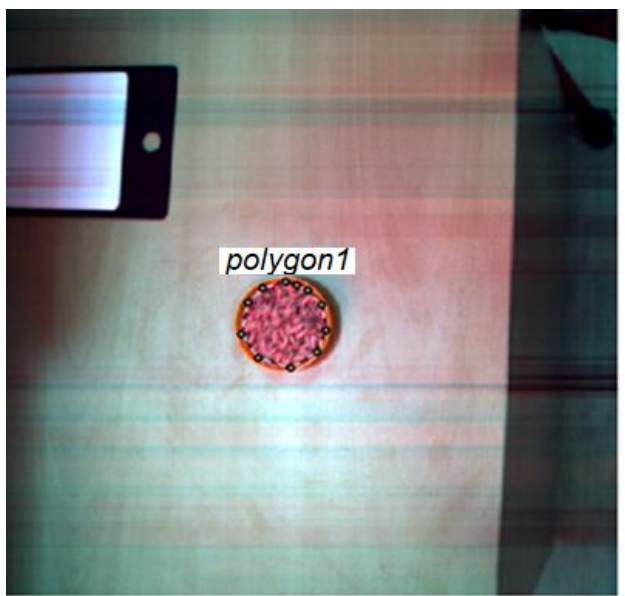

b)

Fig. . Determination of the research area 1 - oat seeds, 2 - calibration plate a) Healthy seeds b) Infected seeds

For the research, 120 hyperspectral images of healthy and infected areas of oats of the variety «ZALP» were taken, the graph shows 12 averaged indicators (Fig. 4). To obtain spectral curves, the images were segmented and the average values of the spectral brightness of the selected segments were used for several images.

\section{Results}

In the graph with the results of calculations (Fig. 4 and Fig. 5), vertical stripes of lilac color are highlighted, which show the content of water and oxygen. Based on this, one can see a significant difference in the graphs in these areas, especially in the areas from 900 to $980 \mathrm{~nm}$. It can be concluded that the content of nutrients in the infected kernel is much less. 


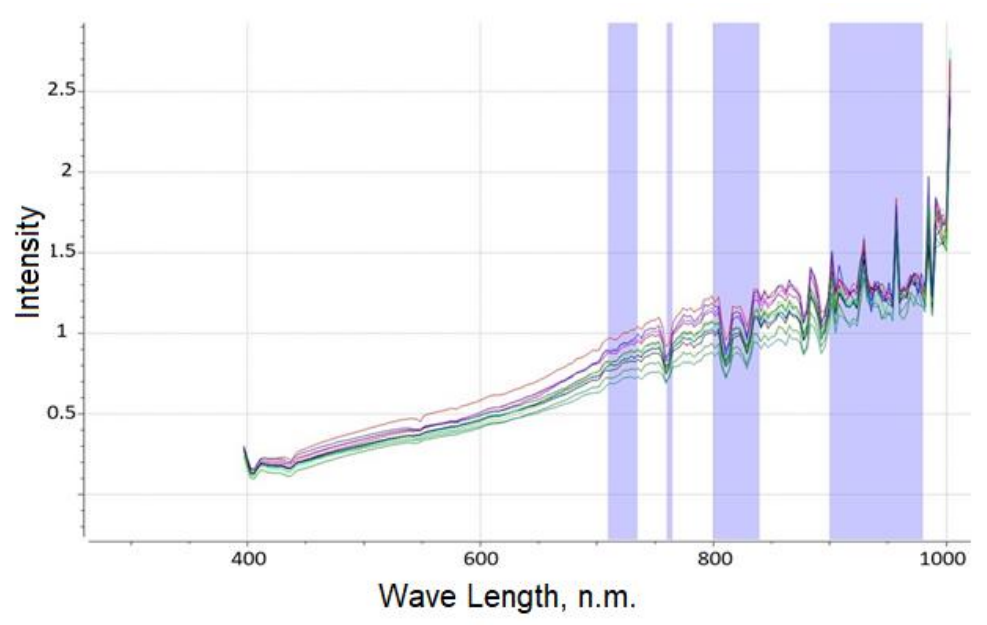

a)

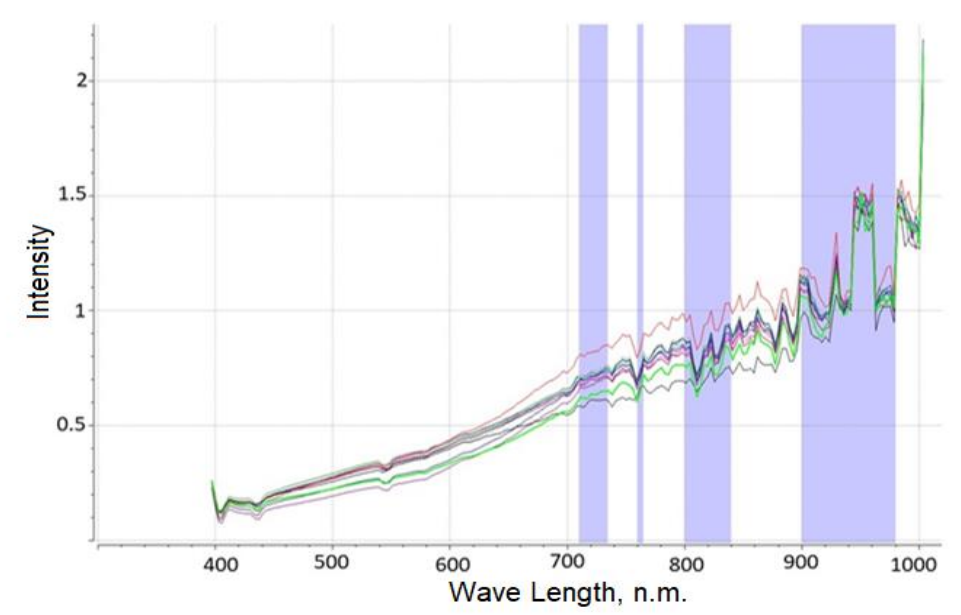

b)

Fig. $\square$. The results of calculating the spectres in the identifying twelve research areas (polygon 1-12) a) the spectrum of infected oats of the variety «ZALP» b) the spectrum of healthy oats of the variety «ZALP».

Also, the entire sample area was highlighted for obtain an average graph for healthy and infected seeds. This makes it possible to examine in more detail the behavior of the graph when comparing healthy and infected seeds (Fig. 5). 


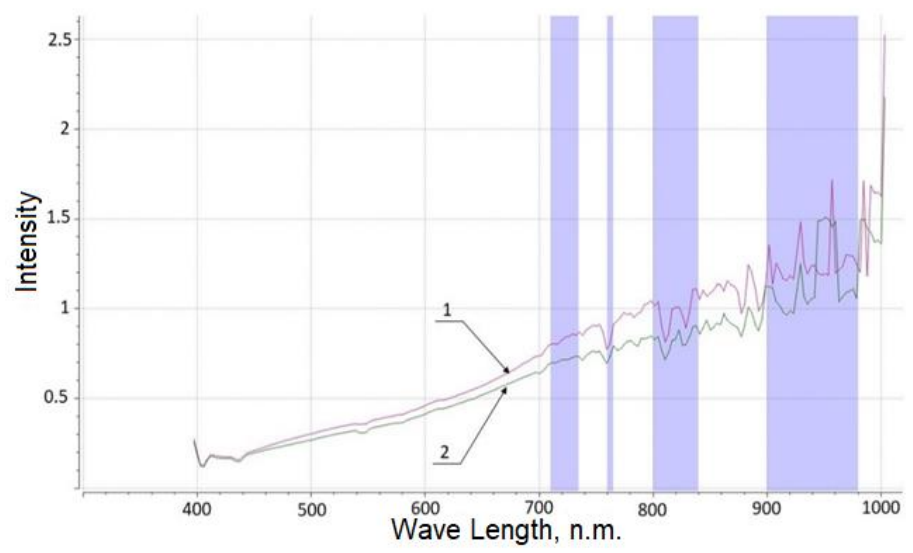

Fig. 5. Comparative results of calculating spectra when selecting the entire reserach area (polygon) 1 - infected oat seeds, 2 - healthy oat seeds

Analysis of the spectral curves obtained for oats of the variety «ZALP» showed that the reflective characteristics of healthy seeds and fusarium infected seeds with differ in the studied parts of the spectrum - visible (400-700 nm) and near infrared (700-1000 nm). Based on the graphs, the general picture of the influence of the disease fusarium on oat kernel is visible. There is a general decrease in the reflectivity of the kernel surface. The strongest deviation of spectral lines is observed in the range from $800 \mathrm{~nm}$ to $1000 \mathrm{~nm}$.

\section{Conclusions}

A method has been developed for identifying saprotrophic and pathogenic microflora by hyperspectral imaging using a Specim IQ camera.

Investigations on a Specim IQ hyperspectral camera revealed the spectral characteristics of seeds of spring oats of the variety «ZALP» infected with pathogenic microflora with toxigenic fungi of the genus Fusarium. Spectral curves in the visible part and horizon 400 $700 \mathrm{~nm}$, as well as in the near infrared range $700-1000 \mathrm{~nm}$ identify the presence of a pathogenic environment and is of great importance for the diagnosis of stress effects on the test material.

The researches carried out have shown the possibility of using the Specim IQ hyperspectral camera for analyzing the reflection spectra of caryopses, both healthy and infected with fusarium. It was possible to identify differences in the spectra of oat seeds, healthy and affected by fusarium. Changes in the spectral characteristics of oat seeds at the border of the visible (400-700 nm) and near infrared regions (700-1000 nm). A Specim IQ hyperspectral camera was used to study two types of biological samples: healthy oat seeds of the variety «ZALP» and infected seeds with toxigenic fungi of the Fusarium genus.

The research was carried out within the framework of agreement No. 075-15-2020-774.

\section{References}

1. H. Bae, Y.-W. Seo, D.-Y. Kim, S. Lohumi, E. Park, B.-K. Cho, J. Korean Soc. Nondestruct. Test. 36, 35-44 (2016)

2. T. Baye, H.C. Becker, J. Am. Oil. Chem. Soc. 81641 - 645 (2004)

3. T.M. Baye, T.C. Pearson, A.M. Settles, J. Cereal. Sci. 43, 236 - 243 (2006) 
4. D.S. Ferreira, J.A.L. Pallone, R.j. Poppi, Food. Res. Int. 51, 53 - 58 (2013)

5. V.V. Alt, T.A. Gurova, O.V. Elkin, D.N. Klimenko, L.V. Maximov, I.A. Pestunov, O.A. Dubrovskaya, M.A. Genaev, T.V. Erst, K.A. Genaev, E.G. Komyshev, V.K. Khlestkin, D.A. Afonnikov, Vavilov Journal of Genetics and Breeding 24 (3), 259-266 (2020)

6. Russian standard GOST 12044-93: Agricultural seeds. Methods for determining the incidence of diseases. - M .: STANDARTINFORM. 64 p. (2011)

7. Phytosanitary examination of grain crops (plant diseases): Recommendations. - $\mathrm{M}$.: FGNU Rosinformogotech. 140 p. (2002)

8. A. Ambrose, L.M. Kandpal, M.S. Kim, W.-H. Lee, B.-K. Cho, Infrared Physics \& Technology 75, 173 - 179 (2016)

9. D. Bohnenkamp, M.T. Kuska, J. Jussila, H. Salo, A.-K. Mahlein, U. Rasche Sensors 18 (2), 441 (2018).

10. M.N. Moskovskiy, Intensification of the process of separation of grain seeds in grain cleaning units. - Rostov-on-Don, 2005. 